\title{
Le « Divin Dali » du visuel au verbal : autoportrait et interaction dans le livre-entretien
}

The "Divine Dali": from the Visual to the Verbal. Self-portrait and Interaction in the Book of Interviews

\section{Ruth Amossy}

\section{(2) OpenEdition}

\section{Journals}

Édition électronique

URL : http://journals.openedition.org/aad/1624

DOI : 10.4000/aad.1624

ISSN : 1565-8961

Éditeur

Université de Tel-Aviv

Référence électronique

Ruth Amossy, « Le « Divin Dali » du visuel au verbal : autoportrait et interaction dans le livre-entretien », Argumentation et Analyse du Discours [En ligne], 12 | 2014, mis en ligne le 20 avril 2014, consulté le 23 septembre 2019. URL : http://journals.openedition.org/aad/1624 ; DOI : 10.4000/aad.1624

Ce document a été généré automatiquement le 23 septembre 2019.

Argumentation \& analyse du discours est mis à disposition selon les termes de la licence Creative Commons Attribution - Pas d'Utilisation Commerciale - Pas de Modification 4.0 International. 


\section{Le « Divin Dali » du visuel au verbal : autoportrait et interaction dans le livre-entretien}

The "Divine Dali": from the Visual to the Verbal. Self-portrait and Interaction in the Book of Interviews

Ruth Amossy

\section{NOTE DE L'ÉDITEUR}

On reprend ici, avec l'aimable autorisation des éditions Transcript, un article publié dans Maurer Queipo, Isabel \& Nanette Rissler-Pipka (éds), Dalís Medienspiele. Falsche Fährten und paranoische Selbstinszenierungen in den Künsten, Bielefeld 2007, pp. 77-93.

1 Salvador Dalí n'a cessé de se mettre en scène tout au long d'une carrière ponctuée de scandales. Cet exhibitionnisme effréné de la part d'un artiste surnommé par Breton Avida Dollars n'a guère été pris au sérieux : les commentateurs, comme le grand public, n'y ont vu qu'une démonstration d'excentricité due à la folie réelle du peintre ou à son sens aigu de la promotion commerciale. Si l'on examine dans leur ensemble tous ses autoportraits visuels et verbaux, on s'aperçoit cependant qu'ils font partie d'un projet artistique soigneusement orchestré. De l'autoportrait pictural aux photographies, des apparitions télévisées aux entretiens écrits et aux textes autobiographiques les plus divers, Dalí poursuit une même entreprise et se réclame d'une même esthétique. C'est dire que chez lui, la tentative d'intégrer l'image de sa personne dans l'espace paranoïaque-critique de l'œuvre s'exprime dans tous les médias en passant incessamment du visuel au discursif ${ }^{1}$.

2 La gageure consistera ici à examiner comme une œuvre paranoïaque-critique de Dalí un livre-entretien quelque peu loufoque réalisé avec Alain Bosquet ${ }^{2}$, pour dégager la contribution singulière de ce genre par rapport à d'autres types d'autoportraits 
daliniens - dont le tableau, la photographie et surtout l'entretien télévisé. Quel est l'apport d'un autoportrait à deux voix d'où l'image visuelle est exclue, où l'interaction constitue la règle du jeu, et dont l'interviewer (Bosquet) et non l'artiste interviewé (Dalí) apparaît comme le maître d'œuvre?

3 Les Entretiens avec Salvador Dali ${ }^{3}$ que surplombe sur la couverture le nom d'Alain Bosquet, lui-même écrivain renommé, soulèvent plusieurs questions. Sans doute est-il clair que L'autoportrait mou avec du lard grillé (1941) ou d'autres autoportraits sur toile font partie intégrante de l'œuvre dalinienne. Leur facture très différente au cours des ans montre bien à quel point l'image de soi, comme tout autre sujet traité en peinture, dépend d'une esthétique. Il suffit pour s'en convaincre de confronter l'Autoportrait mou de la grande période surréaliste avec Autoportrait au cou raphaëlesque de 1922 (La vie publique, 1980 :7), l'autoportrait cubiste de 1923 (ibid: 14), ou Autoportrait (Dalí, nu) de $1954^{4}$. Dans cette optique, la présentation de soi ne diffère pas de la présentation d'une femme, d'une plage ou d'un galet. Il en va de même pour les écrits autobiographiques où la représentation du moi est soumise au style paranoaïque-critique de Dalí : La vie secrète de Salvador Dalí, autobiographie dont la version de 1942 en langue anglaise est illustrée par le peintre, Le journal d'un génie (1964), la Lettre ouverte à Salvador Dalí parue chez Albin Michel en 1966 sont tous de la plume de l'artiste, et remodèlent le genre de l'autobiographie, du journal intime ou de la lettre ouverte au gré de la logique paranoïaque-critique qui est sa marque distinctive. Dans l'autoportrait pictural et le discours autobiographique, le passage de la peinture à l'écriture ne modifie pas la capacité de l'artiste à gérer son autoportrait dans le cadre d'une esthétique personnelle.

Cette position change cependant lorsqu'on aborde les images de Dalí dont il n'est pas l'unique producteur. Que penser des photos où on trouve une mise en scène élaborée correspondant au style familier de Dalí, mais prises, montées et cadrées par d'autres ? On pense bien sûr au Dalí moustache de Halsman, mais aussi à de nombreuses autres photos comme celle de Paul Facchetti (qu'on peut voir dans le catalogue du Centre Pompidou, p.143), ou encore celles de Cecil Beaton dans lesquelles Dalí et Gala adoptent une pose théâtrale derrière deux tableaux daliniens où la silhouette vide d'un homme et d'une femme sert de cadre à un paysage au premier plan duquel apparaît une table couverte d'une nappe. Peut-on considérer que la prise de vue effectuée par un autre est littéralement happée par le génie dalinien dans l'univers paranoïaque-critique duquel elle s'inscrit désormais, et dont la photographie devient le support? Ou plutôt que le photographe apparaît ici comme le collaborateur d'une œuvre collective où Dalí figure à la fois le modèle et le co-producteur? Quoi qu'il en soit, il ressort clairement que la paternité de l'image comme production artistique ne saurait revenir uniquement au photographe : le portrait s'apparente aussi à un autoportrait.

5 Le phénomène se complexifie encore lorsque l'on passe aux interviews télévisées consacrées à Dalí. Tous ceux qui ont vu sur le petit écran des interviews accordées par le maître de la paranoïa-critique ${ }^{5}$ se souviennent de la mise en scène élaborée de sa propre personne qu'il y entreprend : il s'agit bien pour lui de projeter une fois de plus, à l'intention du grand public et donc d'un auditoire peu spécialisé, l'image du fou génial et du grand paranoïaque-critique. Il y parvient par différents moyens, à la fois visuels et sonores. La façon dont il s'apprête, son costume et ses éventuels accessoires, sa moustache, ses poses, ses expressions confortent le public dans une image qu'il connaît déjà. S'y adjoint sa diction très particulière, son accent délibérément outré, son 
discours extravagant où circulent les obsessions répandues dans toute l'œuvre. A cela s'ajoute le fait que la caméra s'attarde souvent sur une toile ou sur le détail d'un tableau qui fait le joint entre la personne mise en scène et son esthétique picturale. Dans la plupart des interviews télévisées, l'interviewer minimise son rôle pour se mettre au service de son sujet - en l'occurrence, la présentation de soi programmée par Dalí luimême. On peut donc avoir l'impression, comme pour les photographies et bien que le genre soit interactif, que l'interview télévisée vient servir une mise en scène gouvernée par les lois de l'univers dalinien, et que c'est de sa fidélité à celui-ci que dépend la réussite de l'émission.

6 On voit donc que l'image visuelle contribue en grande partie à annexer l'interview télévisée au «Dalíland» dans le royaume duquel elle souhaite faire pénétrer le spectateur. Qu'en est-il dès lors de l'interview écrite? Elle ne peut, quant à elle, s'effectuer sur fond des toiles et des dessins de l'artiste, ni plonger à travers eux dans la magie dalinienne. Et cela, même si Bosquet fait précéder chaque séance d'entretiens d'une description minutieuse du décor qui renvoie à l'univers délirant du peintre :

Eparpillées sur les meubles, des pièces en matière plastique sur lesquelles se reflètent, par superpositions, des formes obtenues par des machines électroniques et qui produisent des illusions d'optique peu communes: on a ainsi l'impression d'être devant un miroir très profond avec, de loin, des cercles et des formes ovales... (23).

7 Qui plus est, elle ne donne pas à l'artiste la liberté d'élaborer son image au niveau de la présentation physique de sa personne, avec tous les effets qu'il en tire. Là aussi, la tentative de rendre en mots les effets visuels ne possède pas la force brute dont est dotée la présence corporelle de Dalí dans la photo ou sur l'écran: "Même décor. Présence presque constante de Miodrag Bulatovic [...] Dalí porte un costume qui n'est pas encore terminé, et dont la forme incomplète est cernée de tout un réseau de fils blancs» (81). L'image en première page de couverture, figurant Dalí avec une expression typique aux yeux exorbités, la moustache savamment recroquevillée vers le haut et du même noir corbeau que les cheveux mi-longs, ne suffit pas à compenser ce manque. En bref, contrairement à l'image filmique des interviews télévisées, l'entretien écrit ne donne pas à l'artiste la liberté d'animer et de dominer son image de soi, d'envahir et de régir l'espace où il évolue.

8 Le passage du visuel au verbal soulève dans l'entretien une difficulté supplémentaire. Dans ses textes autobiographiques, le maniement de l'écriture permettait à Dalí d'imposer pleinement son style et ses fantasmes: la gestion de l'œuvre y revenait entièrement à l'auteur. Il n'en va pas de même dans le livre-entretien où le passage à l'écrit est le fait de l'interviewer. La possibilité pour Dalí de maîtriser totalement tous les effets de sa présentation de soi est d'autant plus malaisée que c'est l'interviewer qui a par définition le dernier mot: c'est lui qui rédige le texte et peut le flanquer d'une préface (ce que Bosquet ne manque pas de faire). Cette mainmise effectuée par son partenaire sur sa présentation de soi est lucidement et humoristiquement dénoncée par Dalí lui-même :

Je profite de ce que vous me dites pour me faire un peu de propagande à propos d'un autre livre qui vous fera tous cocus. Albin Michel vient de me commander un ouvrage qui s'appelle «Lettre de Salvador Dali à Salvador Dali ». Là il n'y aura pas d'Alain Bosquet pour s'emparer de moi, et je dirai dans ce livre, beaucoup plus intime, ce que je dois dire à Dali lui-même (31). 

mises en scènes, ses objets, parfois des prises de vue de sa peinture, Dalí s'y trouve également dépouillé de son autorité d'écrivain. Dans cette perspective, on peut se demander comment un livre-entretien peut devenir une partie intégrante de l'œuvre paranoaïque-critique de Dalí en participant de son esthétique.

10 En règle générale, le principe dalinien consiste à s'approprier le medium et le genre dans lequel s'exerce son activité paranoïaque-critique pour le subvertir de l'intérieur et lui imprimer son cachet propre. Or, investir le livre-entretien, c'est avant tout gérer l'interaction qui le définit et le modèle. Rappelons que dans l'interview, et plus particulièrement dans l'entretien d'auteur, la distribution des rôles s'effectue entre d'une part, l'auteur - «l'auteur de l'instant », mais aussi « sa figure mythique, que son interlocuteur lui tend sans arrêt comme une effigie de lui-même, constamment retouchée ", et d'autre part, "l'interlocuteur [...] le représentant d'un certain public dont il se forme une certaine image ». C'est ce public à la fois absent et présent qui est la cible de l'interview - « le dialogue du modèle et de l'interlocuteur n'étant pas un vrai dialogue au premier degré, mais la construction d'un message adressé en commun à un destinataire virtuel » (Lejeune 1980 : 128). Si l'on examine de plus près la dynamique de cette interaction, on s'aperçoit que l'image co-construite de l'auteur y est la résultante "d'une tension entre ce que mettent en place, chacun de son côté, l'écrivain et son interviewer. En effet l'interviewé cherche à se présenter à sa guise tandis que l'interviewer entend le représenter d'une manière qui correspond avec l'objectif de l'entretien » (Yanoshevsky 2006: 165). Cette tension constitutive du genre est pleinement exploitée par Dalí, qui en joue pour réaliser à son gré la mise en scène de sa personne. Et en effet le livre-entretien réalise au plus haut point ce que John Rodden, dans une introduction intitulée "The Literay Interview as Public Performance", appelle «the rhetoric craft of artistic self-fashioning through the form of the literary interview » (Rodden $2001: 1$ ).

11 Quel est l'objectif avoué de l'interviewer? Alain Bosquet, écrivain connu et critique renommé, se doit de dévoiler aux yeux du lecteur une personnalité créatrice exceptionnelle : telle est la vocation de l'entretien avec un artiste célèbre. Dans la mesure où il connaît le peintre de longue date, il est pleinement conscient de la manipulation que Salvador s'apprête à lui faire subir. Il est décidé à l'autoriser dans la mesure où une soumission de bon aloi est indispensable à la bonne marche de l'entretien : sans cela, pas de collaboration, et qui plus est, pas de dévoilement du phénomène Dalí. Aussi fait-il le nécessaire pour assurer la réussite d'un numéro bien rôdé. "Vous vous ramollissez, Dali ", lui dit-il à un moment donné. "A quel point de vue?", s'inquiète Dalí. "Je dis cela pour vous exciter un peu » (82). Ou encore : «Je vous passe vos paroxysmes à vous. Et je joue le jeu... » (56). Face aux excentricités de Dalí, il choisit à son tour un ton provocateur et agressif peu coutumier du genre, qui mime l'irrespect de son interviewé. «Vos réponses ne me satisfont pas », lui lance-t-il cavalièrement (73) ; »Vous m'ennuyez avec votre Corbusier » (49); "Je trouve votre logique aberrante et pleine de trous » (51); ou encore, sur le plan moral (à propos de Lorca): «Je trouve répugnant de parler ainsi de l'assassinat d'un grand poète » (55). Lorsque Dalí affirme qu'impuissance sexuelle et créativité ont partie liée, Alain Bosquet rétorque: «Vous dites cela parce que vous avez soixante ans » (133). Le style de l'échange obéit ainsi au modèle dalinien, créant un climat dans lequel le public pourra 
savourer la singularité de l'artiste. Ce faisant, l'interviewer remplit son mandat, qui consiste à révéler au lecteur la personnalité de Dalí.

Si Bosquet entend par ces choix garder la maitrise de la situation, il ne s'en tient cependant pas là. Sa stratégie consiste en effet à exposer l'excentricité du peintre tout en en minimisant l'importance et en la marginalisant. Il distingue la profondeur de la création artistique de la mise en scène bouffonne du personnage: «En France plus qu'ailleurs, on fait la différence entre vos agissements et votre peinture » (37). Que l'ouvrage tienne à présenter une image de Dalí où triomphe, derrière la figure du clown, celle du peintre génial, se marque bien dans la quatrième de couverture: «Cependant, derrière les paradoxes, les calembours et les provocations de tous ordres, apparaît ce qu'il faut bien appeler "le sérieux" de Dalí, l'extraordinaire passion qu'il voue à son art ». La préface ajoutée à la nouvelle édition de 1983 ("Dalí sans trapèze») se termine significativement par ces mots: "oui, bien sûr, oublions ses ceuvres ratées, qui sont nombreuses, et son action personnelle, qui frise le ridicule et quelque fois le dépasse, par un effroyable masochisme... » (21). En bref, la démarche de Bosquet en tant que légitime représentant non seulement du public, mais aussi de l'institution, consiste à dissocier le "matamore» du génie en soutenant que le premier cache malencontreusement le second:

Ses drôleries, ses pitreries, ses proclamations tonitruantes, ses yeux qui roulent, ses cannes qui fustigent le ciel, ses moustaches qui pourraient empaler les hirondelles ont fait de lui un personnage, sur le compte duquel on se trompe immanquablement (91).

Pour Bosquet, il s'agit en dernière instance de revenir à la tradition du grand homme à laquelle est voué l'entretien d'auteur traditionnel. Ce projet auquel l'interviewer va œuvrer tout au long du dialogue ne convient pas à Dalí, qui entend effectuer et promouvoir la mise en scène paranoïaque-critique de sa personne au même titre que ses autres activités artistiques. Pour réaliser son projet au détriment de celui que poursuit son partenaire, il lui faut déjouer les procédés de celui-ci et reprendre la maitrise d'un texte dont l'interviewer a l'avantage d'être en charge. A l'intention de son interlocuteur immédiat comme de son public, Dalí prend soin d'exposer la façon dont il récupère toute tentative d'annexion de sa personne :

En ce moment même, une demoiselle que je ne connais pas, et qui vient d'entrer ici,
peut-être pour me poser des questions en vue d'un entretien télévisé, s'asseoit près
de nous. Ses genoux sont magnifiques. C'est à partir de là que ma magie va agir.
Plus tard, j'en ferai un élément de ma peinture, une œuvre extraordinaire qui aura
pour base ses genoux et son visage [...] Là est mon génie ! Voyez-vous, tout est dans
cette transfiguration: vous, vous croyez que nous allons écrire un livre ensemble;
elle, elle croit qu'elle va me traîner devant la télévision... Le monde est
constamment cocu (38).

14 Il signifie ainsi à Bosquet qu'il croit le faire participer à ses desseins alors qu'en réalité, c'est Dalí qui l'exploite pour élaborer son œuvre paranoïaque-critique et procéder à la transfiguration artistique dont il a le secret. Le public est dès lors convié à retrouver dans le livre-entretien non seulement les contenus que Dalí entend mettre en valeur, mais aussi la façon dont l'artiste mène la singulière partie où chacun tente de mettre l'autre en échec pour réaliser son propre projet. Telle est en effet la singularité de cet entretien d'auteur en forme de livre : contrairement aux interviews télévisées et aux présentations de soi dont l'image est le support, il permet de dévoiler les modalités de la démarche dalinienne. Il donne à voir, dans le jeu qu'autorise une interaction pleinement maîtrisée, les stratégies au gré desquelles Dalí annexe sa présentation de 
soi à son esthétique propre, faisant participer le public, par les voies du délire et de l'humour critique, à sa "Métamorphose en Narcisse» et, corollairement, à la mythification de sa personne.

Une première stratégie dalinienne consiste à subvertir les règles que l'interview lui impose par le biais du projet de Bosquet, en manifestant qu'il en connaît parfaitement les rouages. Derrière le délire affiché, les commentaires du peintre et la manière systématique dont il se joue des conventions laissent transparaitre un savoir lucide.

Tout d'abord, Dalí caricature le principe du vedettariat qui est à la base de l'interview en présentant une pratique culturelle institutionnalisée en termes de manie privée. Dans la société contemporaine, toute célébrité est censée s'exposer aux yeux du grand public. Cette obligation devient chez Dalí une déviance curieuse, un exhibitionnisme éhonté.

J'ai beau être très connu [raconte-t-il], à Noël, l'année dernière, on m'a offert une petite clochette. Je me promenais dans les rues de New- York, et chaque fois que j'avais l'impression que les gens ne faisaient pas assez attention au Divin Dalí, j'agitais la clochette. Il m'était insupportable de penser qu'on pût ne pas me reconnaître (113).

17 A la question de Bosquet: "Condamné à mort, on vous laisse le choix: quel supplice choisiriez-vous? ", il répond:

Celui qui comporterait le maximum d'exhibitionnisme, et qui me permettrait de me montrer sans cesse ; celui qui comporterait des discours, des harangues, en un mot, celui qui me ferait profiter de l'événement (85).

Plutôt que de se soumettre docilement à la norme de l'interview, Dalí se plaît donc à dévoiler ce qui s'y investit secrètement. Cette première transgression, qui tourne en dérision l'Artiste et ses interviews, met le lecteur en garde : chez Dalí, la présentation de soi est une activité où l'outrance comporte une part de démystification, et où le pitre a le pouvoir de mettre la vérité à nu.

19 Dalí s'en prend, non seulement à la mise en scène des vedettes, mais aussi à ses modalités. Lorsqu'il se présente, l'homme célèbre veille généralement à produire une impression susceptible de renforcer son crédit. Il tient compte des valeurs et des désirs qu'il attribue au public; il prend soin de se conformer au modèle de l'artiste qui circule dans la communauté. Cette règle élémentaire est ostentatoirement violée dans la mise en scène que fait Dalí de sa propre personne. Il ne cesse de choquer le lecteur moyen en foulant aux pieds ses croyances et ses interdits. Dès les premières pages, Dalí déclare qu'étant "un porc excellentissime», il a accepté avec délices la médaille de Franco, vainqueur d'une guerre civile où son ami Federico Garcia Lorca a été mis à mort. Il ajoute qu'il aime la guerre, particulièrement «les costumes de la guerre civile, avec tous les brandebourgs et colifichets. C'est là une image beaucoup plus pétillante, plus picturale, et qui représente la loi» (55). Dalí se définit par ailleurs comme un traître congénital (27), insiste sur sa cruauté (57), son avarice sordide $(25,82)$, explique que, tout en étant monarchiste, il vit à New York parce que «mon éthique personnelle est infaillible. J'habite là où il y a le plus d'argent » (29). Il répète à plusieurs reprises qu'il se " considère comme un peintre très médiocre »- « je crois simplement que je suis meilleur que mes contemporains » (30).

A cela s'ajoutent les réponses incongrues de Dalí, qui déjouent toutes les attentes dans les domaines sur lesquels l'interviewé est censé se prononcer. On se souvient de ses digressions sur l'hibernation, de ses tirades loufoques sur le "pape digestif » et le 
«pape respiratoire» (105), ou sur les Beatniks pour lesquels il s'agit « en se tordant à l'extrême, de faire tomber tous les poils qu'ils peuvent » (56)... Et que penser de ses appréciations peu orthodoxes de Cézanne lequel, ayant passé toute sa vie «à vouloir faire des pommes concaves, n'a réussi à faire que des pommes convexes » (79) ? Toutes les ruptures - défi à la logique, à la tradition, aux idées reçues - sont désormais de règle. Le tout assaisonné de scatologie :

rien de m'est plus néfaste que de travailler de façon appliquée et assidue [...] Même quand je ch... l'attitude immobile paraît superflue : je voudrais que l'on puisse ch... sans aucun effort, de sorte que la m... coulerait comme une espèce de miel fluide, un véritable fil d'Ariane (111).

21 Le premier stade de la stratégie dalinienne consiste donc à briser sciemment tous les automatismes inhérents à un genre et à une situation institutionnelle en violant la décence la plus élémentaire. Cette transgression systématique aux allures délirantes permet de mettre en avant une personnalité dont la folie clamée haut et fort est doublée d'un aspect délibéré et critique: voulue, elle comporte une part de dévoilement et de dénonciation de la norme qui n'est pas sans humour.

Il revient à Bosquet de diriger cette explosion perpétuelle et de maintenir l'artiste dans les limites de son propre projet, celui de l'entretien dont Dalí enfreint et expose les normes. D'un côté, l'interviewer se doit d'enregistrer l'image de soi que projette Dalí, dont les extravagances répétées, incessamment diffusées par les médias, ont d'ores et déjà défini un horizon d'attente particulier. C'est en génie et en fou, en iconoclaste qui ne cesse de violer la morale et le bon sens, que le grand public imagine l'inventeur de la paranoïa-critique, et c'est cette image qu'il désire retrouver dans l'entretien. "Aux yeux d'autrui vous êtes un personnage ", lui dit Bosquet. "Vous êtes un monstre sacré. Vous êtes probablement un monstre» (24). L'interviewer entre donc dans le jeu de l'interviewé en promenant le miroir dans lequel il adopte ses poses favorites. Il souligne l'aspect funambulesque de l'artiste : il qualifie son comportement de « numéro » auquel il se permet d'inviter un ami (67), et qui plus est, de numéro clownesque: « vous ne vous défendez pas d'être un clown » (37). En même temps, il fait entendre une voix critique, qui entend dévaloriser cet aspect du peintre dont il croit nécessaire de minimiser l'importance. Il fait valoir à son interlocuteur qu'il joue sur un effet lassant de répétition dont le choc initial s'est de longue date amorti. De ses agissements, il affirme que « la plupart des gens [...] sont médusés, éblouis pendant une heure, et vite lassés » (37). L'interaction crée ainsi une tension et un retournement. Le démystificateur se voit démystifié : celui qui tourne en dérision toutes les règles du genre que lui propose son partenaire en même temps que toutes les idées reçues, se transforme lui-même dans le discours de Bosquet en stéréotype dérisoire, duquel il est invité à se distinguer.

A ce point précis, cependant, se dévoile un autre aspect de la stratégie de Dalí. C'est précisément la stéréotypie et son effet lassant de répétition qu'il récupère à son profit. En effet, le «mythe » de Dalí se nourrit de la mise en place et de la réduplication à l'infini d'une image figée. Dalí se fonde une fois de plus sur la connaissance précise d'un mécanisme culturel contemporain, celui de la «mythification » au sens des Mythologies de Roland Barthes, pour l'exploiter sans scrupules. Sur le marché où l'on ne cesse de consommer du mythe, seules accèdent à ce rang les figures qui, comme Marilyn Monroe, James Dean ou Charlot, ont été réduites à une image simple et figée qui se grave aisément dans l'imagination populaire. La schématisation et la récurrence sont la condition sine qua non du processus: c'est lorsqu'elle est toujours la même, reconnaissable entre toutes et incessamment redupliquée, qu'une image s'incruste dans 
l'imaginaire d'une époque (Amossy 1991). Dalí ne se soucie donc guère des dangers de la stéréotypie dont l'avertit son partenaire. Tout au contraire, il prend soin de se mettre en scène sous les traits d'un personnage stéréotypé; tout un style, comprenant les moustaches agressives, les effets d'yeux et de voix, le comportement loufoque, est mobilisé à ces fins. Ne se présente-t-il pas lui-même comme un personnage de la Commedia dell'Arte, un "Arlequin » (37) ? Comme de nos jours, les médias contribuent massivement à la mythification des êtres et des choses, Dalí veille également à ce que son image soit constamment diffusée dans les journaux et à la télévision. "Quels effets lvous font les attaques dans la presse? ", lui demande Bosquet, et Dalí de rétorquer : « Elles sont toujours réconfortantes. On parle de Dalí sans arrêt... » (116).

Sans doute ne suffit-il pas de faire circuler sans relâche un stéréotype pour le hisser au rang de mythe: il faut également qu'il soit fondé en valeur et se donne comme une image fascinante et un modèle idéal (Amossy 1991). Là encore, Dalí reproduit fidèlement un mécanisme culturel dont il a démonté les rouages. Avec une différence, cependant: c'est qu'il produit lui-même un processus qui s'impose en général à partir de son dynamisme propre. J'entends que Dalí se divinise lui-même dans une entreprise bouffonne et cynique qu'il ne prend pas la peine de voiler. "Vous vous appelez, le divin Dali' vous-même? " (24) demande Bosquet, et plus loin : «ce Dali divinisé à bon marché par lui-même existera-t-il vraiment?»(50). En fait, Dalí reprend l'imagerie populaire de l'artiste comme génie et comme fou en la portantà son paroxysme. «Le Divin Dali n'a pas d'égal » (113) déclare péremptoirement celui qui affirme la «structure impérialiste de son propre génie » (35). Et d'annoncer :

Je vous apprends là une nouvelle qui est encore secrète : le Divin Dali est en train de récrire complètement le petit Larousse [...] Dans quelques années j'aurai mon Larousse à moi et le monde pourra apprendre ce que je pense de chaque chose... (116)

En travaillant à l'édification de son mythe, Dalí n'ignore pas qu'elle ne contribue guère à la compréhension de l'œuvre. A la limite, elle acquiert dans la conscience populaire un statut indépendant de la connaissance de l'art pictural. Lorsque Bosquet objecte à Dalí que les jeunes d'aujourd'hui le prennent pour un mage, et ignorent son œuvre, le peintre rétorque :

C'est vous que cela gêne. Moi, ce qui me fait plaisir, c'est que des gens très quelconques, qui ne comprennent rien, peuvent me dire «Bonjour, Salvador ». Il m'importe assez peu qu'on me prenne pour un peintre, un homme de la télévision ou un écrivain. Le principal, c'est qu'il y ait un mythe Dali, même incompris et même entièrement faux (123).

Là où Bosquet essaye de ramener Dalí à ses vues en lui prouvant la vanité d'une mise en scène clownesque qui nuit à la reconnaissance de son art, Dalí déjoue toute mainmise sur sa présentation de soi et réitère la primauté de son propre projet - au sein duquel la mise en scène délirante du moi est primordiale. L'excentricité le pose délibérément en fou, tandis que l'outrance délibérée à vocation démystifiante dévoile le critique lucide et l'entreprise dûment programmée.

Dans le jeu où l'entraine Dalí, le public assume simultanément deux positions quasi incompatibles. D'un côté, il tient le rôle du "cocu», celui dont Dalí ne cesse de se moquer. Ainsi Dalí explique qu'il a créé une manière spéciale de parler l'anglais, en roulant les $r$ comme en espagnol et en exagérant sa prononciation française :

très souvent, ni les Américains ni les Anglais ne comprennent ce que je dis, mais s'il leur arrive de saisir un petit détail, il en résulte un tonnerre d'applaudissements. Le 
phénomène est extraordinaire ; au fond, ils s'applaudissent eux-mêmes de m'avoir compris (144). consentantes en leur dévoilant le mécanisme du piège qu'il dresse à leur intention. Il met le lecteur - le rieur - de son côté par la mise à nu parodique de ses propres procédés. Il en fait un complice dès lors qu'il lui offre en pâture le commentaire lucide de la méthode adoptée. Et en effet, en dévoilant la façon dont il exploite en s'en jouant les normes de la présentation de soi et de l'interview, Dalí associe le public à son entreprise de mystification. Il expose sans fard la démarche au gré de laquelle il édifie son propre mythe. Il veille également à mitiger cette tentative de déification pour le moins étonnante par un humour constant. Il décrète ainsi, à propos d'un procès perdu : «Je suis un être suprême, il va falloir que je m'adresse à la Cour suprême. Chez moi tout va au degré le plus haut, c'est-à-dire à la Cassation » (143). Ou encore :

Les obsessions sexuelles sont le fondement de la création artistique. Les insatisfactions accumulées mènent à un processus que Freud appelle celui de la sublimation [...] les gens portés sur l'amour physique ne font rien du tout: ils s'expriment par le f... un peu partout. Pour le Divin Dali, si par hasard il y a une goutte de f... qui sort, tout de suite il a besoin de la rentrée d'un chèque de grande valeur, afin que la dépense soit immédiatement remboursée. Mais comme cela arrive très rarement, tout se transforme en œuvres d'art, en fonctions spirituelles... (133).

Dans cette perspective, le livre-entretien offre un avantage qui le singularise parmi les autres pratiques daliniennes de présentation de soi. Dalí y noue avec ses allocutaires un rapport complexe dont il lui fait savourer lui-même toutes les nuances. C'est qu'il lui demande d'apprécier l'opération de «crétinisation » dont il fait l'objet en l'en rendant complice; il s'adresse à son intelligence non seulement en démontant à ses yeux sa machine à décerveler ubuesque, mais aussi en lui présentant un discours truffé d'allusions dont les défis à la tradition culturelle impliquent une grande familiarité avec celle-ci, et qui ne peut être saisi pleinement que sur fond d'art dalinien. Dès lors qu'il est invité à un spectacle de marionnettes dont on lui dévoile les ficelles, le public doit être enclin à applaudir.

Ainsi donc, la stratégie dalinienne prévoit, pour sa bonne marche, deux réactions opposées et complémentaires. La première, de pur refus, est sensible à la provocation à laquelle elle confère par là même sa force de choc. Le lecteur moyen comme le destinataire cultivé s'y rejoignent dans une même indignation, ou dans la même incompréhension moqueuse. "Avez-vous peur que les jeunes se moquent de vous?",

Argumentation et Analyse du Discours, 12 | 2014 
demande Bosquet, et Dalí de répondre: "Je le désire au contraire. Les moqueries prouvent que je les domine » (96). La seconde réaction, réservée à l'élite (et qui ne veut se définir comme élite ?) stipule une reconnaissance lucide et une participation active au jeu dalinien. Elle allie l'admiration et le sarcasme. "Vous acceptez qu'on vous admire avec sarcasme?" demande Bosquet, et Dalí répond: "avec le plus de sarcasme possible » (114). A ceux qui assimilent les règles du jeu avec le détachement ironique et la passion qui conviennent, l'artiste offre les plaisirs de l'humour et de la lucidité.

Il leur propose ainsi, à l'instar du dandy, un objet d'art qui est sa propre personne. Ce mélange d'humour et de désinvolture à propos de tout et de lui-même, Dalí l'explique en effet par le dandysme :

- Peut-on traverser la vie en ayant l'air de se moquer de tout?

- Puisque c'est mon cas ! Et c'est le cas de tous les dandys qui réussissent (89).

L'une des clés de la méthode dalinienne est dans cette notion de dandysme, qui assied la supériorité sur une présentation théâtrale soigneusement apprêtée, un ensemble de règles appliquées à la mise en scène de sa propre personne. Car le dandy, ne l'oublions pas, joint le sérieux et le futile; il affirme la supériorité de son esprit par son comportement extérieur. Et en effet, que fait Dalí sinon se transformer lui-même en objet d'art ? Un objet semblable à ceux qu'il s'est toujours complu à fabriquer, agressif, relevant à la fois du grotesque et du sérieux, de la folie et de la lucidité; un objet surréaliste étrangement proche des mannequins qu'il avait disposés dans les vitrines de la $5^{\mathrm{e}}$ Avenue. La mise en scène du moi obéit bien chez Dalí aux règles de son esthétique. Elle viole les tabous non seulement pour provoquer le bourgeois, mais aussi pour remonter aux sources fécondes de l'inconscient et produire une image fantasmatique dans laquelle le symbolisme onirique se mêle à l'humour. Comme dans la méthode paranoïaque-critique où le tableau, contemplé à distance et avec un certain recul, dévoile une image qui échappait à la première observation, les tirades de Dalí permettent de voir, derrière l'image du fou qui se singularise par ses excentricités, la figure de l'artiste qui joue sciemment de la transgression pour dévoiler le monde des fantasmes. Exhibitionnisme, érotisme, scatologie : chaque domaine abordé par Dalí se soumet au même jeu de métamorphoses, changeant de forme au gré du recul et du degré d'attention apportés. La scatologie, sujet controversé par excellence, le montre bien. Elle se donne chez Dalí comme une provocation outrancière qui choque jusqu'aux meilleurs esprits. Elle est aussi, néanmoins, hommage au bas corporel qui est à la source d'un art fécond (et en particulier du grotesque). Simultanément, elle autorise le dévoilement impudique des images de l'inconscient refoulées par les convenances. A tous ces titres, la scatologie participe tant du discours dalinien que de l'univers de ses toiles - comme le montre bien le tableau intitulé Jeu lugubre.

Ainsi l'univers où se meut Dalí devient l'espace où les images de soi, comme celles de l'autre ou des objets, ne cessent de se métamorphoser en abolissant toutes les frontières entre le monde du fantasme et celui de la réalité. C'est là, justement, la particularité de la représentation dalinienne : l'obsession y revêt des contours précis, l'irrationalité y est « concrète » et la paranoïa " critique ». De même, la folie y est génie, le ludique sérieux - et inversement. A Bosquet qui demande : "Où est la limite entre le génie et la folie? ", l'artiste répond:

Je suis non seulement un agent provocateur, mais aussi un agent simulateur. Je ne sais jamais quand je commence à simuler ou quand je dis la vérité. Cela est caractéristique de mon être profond [...] Il faut en tout cas que le public ne sache 
point si je rigole ou si je suis sérieux; de même, il ne faut pas que je le sache moimême (98). le spectateur, qu'à révéler la relation complexe du sujet à son moi. Celui-ci se définit comme la persona projetée dans la réalité, le masque sans cesse refaçonné ; c'est celui de l'Arlequin et du saltimbanque qui se moque de lui-même et des autres dans une société commercialisée vouée à une publicité tapageuse. C'est aussi un être scindé dont la réunification utopique ne peut s'effectuer que dans la mascarade où s'exhibe un Dalí à plusieurs têtes (c'est le costume que portait Dalí au bal onirique de 1941). "Vous savez que la structure de ma personnalité est toujours binaire, je suis bicéphale et double » (45), dit Dalí à Bosquet. De même les réponses de Dalí, en mêlant la profondeur et la pitrerie, dessinent cette figure pour le moins double qui se donne à elle-même la réplique, et où chaque élément se prête à des interprétations divergentes. œuvre en le marquant du sceau de la paranoïa-critique. Il le fait en soumettant son image de soi, et le genre de l'entretien dans l'espace duquel elle s'édifie, aux lois d'une esthétique hautement revendiquée. Il faut souligner, cependant, que s'il entraîne l'interview dans la ronde carnavalesque de la transgression, il n'en sape pas pour autant les fondements. Si la réduplication dérisoire de la pratique sociale en dévoile le caractère réglé, conventionnel et arbitraire, c'est pour l'ériger en jeu, c'est-à-dire pour permettre à tous les participants de continuer à jouer. La gestion à la fois délirante et lucide de l'interaction ludique est le grand atout du livre- entretien.

C'est dans la collaboration tout en tensions et rebondissements des deux partenaires, dans la co-construction d'une image de l'artiste à l'intention du public et dans la relation singulière que Dalí met en place avec son lecteur, que se marque la spécificité et l'apport de ce genre par rapport aux autres modalités de l'autoportrait dalinien. Il permet en effet l'intégration de la personne de l'artiste à son œuvre et sa mytification par des moyens qui diffèrent de ceux du tableau, de la photographie et qui plus est, de l'interview télévisée. Sans doute, la maitrise de l'artiste qu'autorisent le tableau ou l'autobiographie y cède la place à un partage des pouvoirs. Mais la manipulation de l'interaction permet de compenser cette perte d'autorité - comme d'ailleurs de substituer à la force de la présence corporelle un pouvoir d'un autre ordre. Dans la joute verbale dont Bosquet se fait le scribe, Dalí s'exerce à investir à la fois le dialogue dont se nourrit l'interview, et l'image de soi qui s'y édifie. Les stratégies qu'il déploie à cet effet dépassent la simple présentation du grand paranoïaque-critique: elles montrent les secrets de sa fabrication. Contrairement à ce qui se produit dans les productions visuelles, y compris dans l'interview télévisée où l'artiste joue de sa présence physique et profite des éléments picturaux filmés, le livre-entretien permet de rendre le public complice du travail de déconstruction et de mythification dont se nourrit la présentation de soi. Il se dote d'une sorte de métadiscours qui dévoile les modalités de la mise en scène au moment même où elle s'effectue. Ce n'est pas le moindre intérêt de ce livre-entretien que Dalí travaille à intégrer dans le monde à la fois délirant et critique dont il détient la clé. Le nom de Bosquet a beau surplomber l'ouvrage, le livre ne s'en inscrit pas moins dans la série des oeuvres daliniennes - sans doute dans la catégorie de celles qui se construisent à plusieurs en faisant entrer les partenaires dans l'espace délirant, grotesque et lucidement programmé du Maître.

Argumentation et Analyse du Discours, 12 | 2014 


\section{BIBLIOGRAPHIE}

Dalí, Salvador. 1935. La Conquête de l'Irrationnel (Paris : Editions surréalistes)

Dalí, Salvador. 1942. The Secret Life of Salvador Dali (New York : Dial Press)

Dalí, Salvador. 1964. Journal d'un génie (Paris : La Table ronde)

Dalí, Salvador. 1966. Lettre ouverte à Salvador Dalí (Paris : Albin Michel)

Dalí, Salvador.1971. Oui. Méthode paranoïaque-critique et autres textes1971 (Paris : Denoël/Gonthier)

Dalí, Salvador. 1979. Oui. Méthode paranoïaque-critique et autres textes, tome II (Paris : Denoël)

Dalí, Salvador. 2006. La vie secrète de Salvador Dalí : suis-je un génie ? Edition critique établie par Frédérique Joseph-Lowery (Lausanne : L'Age d'Homme)

Abadie, Daniel. 1980. La vie publique de Salvador Dalí [Centre Georges Pompidou, 18 decembre 1979-21 avril 1980 : catalogue de l'exposition] Ades, Dawn. 1984. Dalí (London/New York : Thames and Hudson)

Amossy, Ruth. 1995. Dalí ou le filon de la paranoïa (Paris : PUF)

Amossy, Ruth. 1991. Les idées reçues. Sémiologie du stéréotype (Paris : Nathan)

Bosquet, Alain. 1983. Entretiens avec Salvador Dali suivis de La Conquête de l'Irrationnel (Paris : Belfond)

Finkelstein, Haim. 1996. Salvador Dali's Art and Writing, 1927-1942, Cambridge, Mass. : Cambridge U. Press.).

Gérard, Max. 1974. Dalí.. Dalí.. Dalí... (Paris : Draeger)

Lejeune, Philippe 1980. «L'entretien radiophonique », Je est un autre. L'autobiographie de la

littérature aux médias (Paris : Seuil)

Reynolds, A. Morse. 1974. Salvador Dalí, A Panorama of his Art (Cleveland, Ohio :The Salvador Dalí Museum)

Rodden, John. 2001. Performing the Literary Interview. How Writers Craft Their Public Selves (Lincoln/ London : U. of Nebraska Press)

Yanoshevsky, Galia. 2006. Les discours du Nouveau Roman. Essais, entretiens, débats (Villeneuve d'Ascq : P. U. du Septentrion)

\section{NOTES}

1. Ce travail poursuit la réflexion entamée dans Ruth Amossy 1995. Dali ou le filon de la paranoïa (Paris : PUF), qui étudie la façon dont divers genres - comme l'autobiographie ou l'essai critique sur l'Angélus de Millet - sont annexés à l'esthétique paranoiaque-critique de Dalí qui en opère une véritable métamorphose.

2. Je n'exposerai pas ici la théorie bien connue de la paranoia-critique de Dalí, dont la théorisation a été faite dans les textes recueillis dans les deux volumes de Oui 1 et Oui 2, et dont de nombreux commentaires ont été offerts. Voir en particulier Reynolds 1974 ; Ades 1984 ; Amossy 1995; Finkelstein 1996.

3. Toutes les citations dans le texte renvoient à l'édition Belfond (Bosquet 1983). 
4. Collection privée, Memphis. On trouvera une reproduction dans Gérard 1974 : illustration 98.

5. On peut, à titre d'exemples, consulter les interviews télévisées qui se trouvent sur le site de l'INA.

\section{RÉSUMÉS}

Cet article examine la façon Salvador Dali se met en scène dans un livre-entretien d'où disparaît la dimension visuelle de son autoportrait, et où l'interaction avec l'interviewer ne lui permet pas de gérer librement sa présentation de soi. On peut voir comment le projet de l'interviewer - faire un portrait du grand homme en acceptant mais en minimisant ses excentricités - se trouve en butte à celui de l'interviewé qui veut accentuer la dimension délirante de sa performance et faire de l'entretien une partie intégrante de son œuvre paranoïa-critique. L'une des stratégies de Dali jouant à contrer son interviewer consiste à exhiber comiquement les règles du genre dans une série de transgressions spectaculaires qui le posent en fou et en critique tout à la fois. Une stratégie complémentaire consiste à outrer le stéréotype de l'artiste en génie et en fou pour contribuer à une image de sa personne qui sert un projet à la fois mercantile et esthétique (paranoïa-critique). Ainsi, à travers un délire et un humour maîtrisés, l'interviewé tente de contrer son interviewer, faisant participer son public à sa Métamorphose de Narcisse et à la mythification de sa personne.

This essay explores the self presentation of Salvador Dali in an interview book in which the visual dimension of his self-portrait disappears, and where the interaction with the interviewer does not allow him to freely stage his own self. The project of the interviewer - to exhibit the great man by tolerating but minimizing his excentricities - clashes with the project of the interwiewee, who wants to reinforce the delirious dimension of his performance, and fully integrate the interview in his paranoia-critical work. One of Dali's strategies consists of revealing in a comic light the rules of the genre in a series of spectacular transgressions depicting him as both a madman and a critique. Another, complementary strategy consists of an exacerbation of the stereotype of the artist as a mad genious in order to contribute to a self-image serving a project both mercantile and esthetic (paranoïa-critical). Thus through critical delirium and humor, the interviewee tries to undo his interviewer, and tranform his audience into a participant of his Metamorphosis of Narcissus and the mythification of his person.

\section{INDEX}

Mots-clés : Dali (Salvador), interaction, livre entretien, paranoïa-critique, présentation de soi

Keywords : Dali (Salvador), interaction, interview book, paranoia-critique, presentation of self

\section{AUTEUR}

\section{RUTH AMOSSY}

Université de Tel Aviv, ADARR 